\title{
The Impact of an Enhanced Interpreter Service Intervention on Hospital Costs and Patient Satisfaction
}

\author{
Elizabeth A. Jacobs, MD, MPP ${ }^{1,2}$, Laura S. Sadowski, MD, MPH ${ }^{1,2}$, and Paul J. Rathouz, PhD ${ }^{3}$ \\ 'Collaborative Research Unit, John H. Stroger Jr. Hospital of Cook County, Chicago, IL, USA; ${ }^{2}$ Division of General Internal Medicine, Rush \\ University Medical Center, Chicago, USA; ${ }^{3}$ Department of Health Studies, University of Chicago, Chicago, USA.
}

\begin{abstract}
BACKGROUND: Many health care providers do not provide adequate language access services for their patients who are limited English-speaking because they view the costs of these services as prohibitive. However, little is known about the costs they might bear because of unaddressed language barriers or the costs of providing language access services.
\end{abstract}

OBJECTIVE: To investigate how language barriers and the provision of enhanced interpreter services impact the costs of a hospital stay.

DESIGN: Prospective intervention study.

SETTING: Public hospital inpatient medicine service.

PARTICIPANTS: Three hundred twenty-three adult inpatients: 124 Spanish-speakers whose physicians had access to the enhanced interpreter intervention, 99 Spanish-speakers whose physicians only had access to usual interpreter services, and 100 English-speakers matched to Spanish-speaking participants on age, gender, and admission firm.

MEASUREMENTS: Patient satisfaction, hospital length of stay, number of inpatient consultations and radiology tests conducted in the hospital, adherence with follow-up appointments, use of emergency department (ED) services and hospitalizations in the 3 months after discharge, and the costs associated with provision of the intervention and any resulting change in health care utilization.

RESULTS: The enhanced interpreter service intervention did not significantly impact any of the measured outcomes or their associated costs. The cost of the enhanced interpreter service was \$234 per Spanishspeaking intervention patient and represented $1.5 \%$ of the average hospital cost. Having a Spanish-speaking attending physician significantly increased Spanishspeaking patient satisfaction with physician, overall hospital experience, and reduced ED visits, thereby reducing costs by $\$ 92$ per Spanish-speaking patient over the study period.

CONCLUSION: The enhanced interpreter service intervention did not significantly increase or decrease hospital costs. Physician-patient language concordance reduced return ED visit and costs. Health care providers need to examine all the cost implications of different language access services before they deem them too costly.
KEY WORDS: language barriers; interpreter services; hospital costs; patient satisfaction.

J Gen Intern Med 22(Suppl 2):306-11

DOI: $10.1007 / \mathrm{s} 11606-007-0357-3$

(C) Society of General Internal Medicine 2007

$\mathrm{T}$ he conversation between physician and patient has long been recognized to be of diagnostic import and therapeutic benefit. Unfortunately, many patients in the United States cannot benefit from this fundamental interaction because of language barriers. According to Census 2000, more than 46 million people in the United States do not speak English as their primary language and more than 21 million speak English less than "very well". ${ }^{1}$ Many of these residents do not receive needed health care or the standard of care because most health care organizations provide inadequate interpreter services. $^{2-8}$ Many health care providers do not provide adequate interpretation because of the perceived financial burden. ${ }^{9,10}$ However, they neglect to take into account the cost of the consequences of failing to provide adequate interpretation or the potential benefits of improving communication with patients. This may be due in part to the paucity of data documenting these costs and benefits.

We know of only 6 studies to date that have directly set out to measure these costs and benefits. Two studies found that the use of ad hoc interpreting by employees has an opportunity cost for institutions in the form of staff time lost to interpreting. ${ }^{11,12}$ Another study, in a pediatric emergency department (ED), found that the presence of a language barrier between physician and parents accounted for a \$38 increase in charges for testing and a 20-minute longer ED stay compared to encounters in which there were no language barriers. ${ }^{13}$ Three additional studies have investigated the direct costs and potential cost-savings of providing professional interpreter services. They have found that the cost of these services is quite low relative to most health care costs ${ }^{14}$ and that they can reduce the cost of care provided in the $\mathrm{ED}^{15}$ and follow-up visit charges after ED evaluation. ${ }^{16}$ There is still a need for investigations that compare the costs of providing adequate linguistic access services to the cost of not providing them, especially in the inpatient setting. 
Using a conceptual model developed by the U.S. Department of Health and Human Services' Office of Minority Health to guide research efforts about the costs of language barriers in health care, ${ }^{17}$ we undertook a study to (1) provide data on the costs of failing to provide adequate interpreter services and (2) measure the direct costs and cost-offsets of enhanced interpreter services use in the care of Spanish-speaking hospitalized patients. We define adequate interpreter services as those provided by trained and tested interpreters available on-site in a timely manner. Our overall hypothesis was that hospitalized Spanish-speaking patients who cannot readily and adequately communicate with their providers would generate higher inpatient costs compared to those who are able to communicate with their clinicians through the assistance of readily available, trained professional interpreters. Our secondary hypotheses were that Spanish-speaking patients who cannot readily and adequately communicate with their clinicians will be less satisfied with their hospital stay and physician-patient communication; will have higher rates of post discharge ED utilization and hospitalization; and will have poorer adherence with scheduled outpatient visits than patients who had the assistance of a readily available, professional interpreter.

\section{METHODS}

\section{Setting}

The study took place from January 19, 2005 to June 30, 2005 and involved the Internal Medicine service of a large public hospital in the City of Chicago, IL. The Internal Medicine service is organized into 3 firms; the firms consist of equal numbers of attending and resident physicians who work together, on a monthly rotating basis, to care for patients assigned to their firm when they are admitted to the hospital. In any given month, each firm has 4 teams of 5 physicians each (1 attending, 2 residents and 2 interns) who admit patients to the hospital every fourth night. Patients admitted to the medicine service of the hospital are assigned to be under the care of 1 of the 3 firms based on which team is next in line for an admission. This assignment is nonpurposeful and based only on the order of admission. Patients are admitted to the next available hospital bed so there are no geographical firm boundaries.

Two of the 3 firms were randomly selected to participate in the study and an enhanced interpreter intervention was randomly assigned to 1 of these 2 firms. We did not randomize patients to receive the interpreter intervention because it would have been logistically difficult for the interpreters to work across firms and we did not want the intervention to influence the physician practice for those patients not assigned to receive the intervention.

\section{Study Participants}

All patients who were admitted to the study firms during this time period and had a Hispanic surname, or were identified as needing an interpreter by hospital staff were approached by bilingual research staff. These patients were invited to participate if they were 18 years of age or older and stated that they spoke only Spanish or had difficulty communicating in a language other than Spanish. We also recruited English speaking (ES) patients admitted to the study firms and matched them to Spanish-speaking participants on gender, age, week of admission, and firm. ES patients were used to control for firm effects not related to the enhanced interpreter services intervention. Patients were excluded from participation in the study if they were unable to consent to participation because of cognitive or mental impairments. All participants received \$20 upon discharge from the hospital. The Institutional Review Board of the Cook County Bureau of Health Services approved the study.

\section{Enhanced Interpreter Service Intervention}

The enhanced interpreter service intervention consisted of 2 trained Spanish medical interpreters assigned to work with Spanish-speaking patients and their caregivers throughout their hospital stay. Both interpreters graduated from a yearlong, intensive, community college interpreter training program including instruction on medical vocabulary, standards, ethics of interpreting, patient confidentiality, and triadic communication. Both completed a 120-hour internship. A single interpreter was available 7:30 A.M. to 3:30 P.M. daily during the study period. One interpreter was available Monday through Friday and the other was available during the weekend. The interpreters rounded with the intervention firm physicians each morning and were paged by the physicians, nurses, and patients when needed. The chart of each Spanishspeaking patient cared for by the intervention team had a note alerting all staff of the availability of the interpreter.

Spanish-speaking patients cared for by the nonintervention team who spoke Spanish received the usual care: no interpretation or use of ad hoc interpreters (family, friends, and untrained bilingual staff), telephonic interpreters, or the usual hospital interpreter service. The usual hospital service is not sufficiently staffed to meet the current demand for Spanishspeaking interpreters, resulting in significant delays (sometimes up to several hours) between requesting an interpreter and arrival of the interpreter at a patient's bedside. As a result, clinical staff frequently relies on ad hoc interpreters to communicate with their Spanish-speaking patients. In addition, not all hospital interpreters are trained, and those that are had more limited training than the intervention interpreters.

\section{Measurements}

Baseline patient variables included age, gender, country of birth, years lived in the United States, language ability, education, marital status, household income, and numbers of times they had seen a physician or had been hospitalized in the past year. All participants were asked in what language they usually read, thought, and spoke and chose from the following response categories; only Spanish, Spanish better than English, both equally, English better than Spanish, or English only. Education was categorized as elementary school or less, middle school, some high school, high school or GED, or greater than high school. Insurance status was categorized as private insurance, Medicare, Medicaid, no insurance, or other. Marital status was categorized as single/never married, married, not married but living with a committed partner, or separated/ divorced/widowed. Household income was categorized as $<\$ 10,000, \$ 10,000-24,999$, and $\$ 25,000$ or more. Diagnoses 
included in the patient's electronic medical record (EMR) discharge summary were used to calculate the Charlson comorbidity index (CCI) scores for each participant. ${ }^{18}$

The following outcome variables were abstracted from the EMR. Length of stay (LOS), number of specialty consultations and radiology tests during the hospital stay, ED visits and hospitalizations in the 3 months after hospital discharge, and adherence to follow-up outpatient visits scheduled at discharge. Patients were considered adherent if they went to at least 1 follow-up visit.

Satisfaction with the hospital stay was measured using the Hospital Consumer Assessment of Healthcare Providers and Systems (H-CAHPS), a previously-validated 24-item instrument available in English and Spanish. ${ }^{19}$ Participants completed the survey at discharge with the help of the research assistant (RA) or, if they were discharged outside of the RA's working hours, completed it over the phone in response to RA questioning, or mailed it in. We analyzed items from the $\mathrm{H}-$ CAHPS that would likely be affected by communication, including satisfaction with nursing care (4 items), satisfaction with physicians ( 4 items), and overall satisfaction with the hospital stay (2 items).

We also collected information on attending and resident physicians' Spanish fluency. We tested the physicians who indicated that they spoke Spanish with hospitalized patients and who had not trained in or practiced in a Spanish-speaking country. Physicians deemed proficient on a test modeled on the American Council on the Teaching of Foreign Languages' oral proficiency interview, ${ }^{20}$ and physicians who had trained in or practiced in a Spanish-speaking country were included in the "Spanish-speaking physician" category.

Costs were calculated using the average costs of care provided at the study institution in 2000 , the most current year for which this information was available, and the costs of providing the interpreter service intervention during the study period. Both included overhead costs. The intervention costs include interpreter salaries, which were constant throughout the study, regardless of how many encounters were interpreted each day.

\section{Statistical Analyses}

Analyses were conducted to test our hypotheses that LOS, inpatient service utilization, and post discharge events would be lower and post discharge follow-up and satisfaction higher for Spanish-speaking patients whose physicians had access to the intervention (SS-I) compared to Spanish-speaking patients whose physicians had access only to usual care (SS-U). For each outcome, we fitted regression models with variables for Spanish-speaking patient group (SS versus ES), for firm (intervention versus usual care), and for their interaction. The interpreter services effect is given by the interaction, as it measures the difference between the effect of the firm for the SS (SS-I versus SS-U) and for ES patients, thereby isolating differences between SS-I and SS-U attributable to the enhanced interpreter services intervention. Demographic variables were included for adjustment, as was an indicator for SS patients having a Spanish-speaking attending physician. For those outcomes with a significant Spanish-speaking attending effect, a second model was fitted with the interaction between the interpreter services intervention and Spanish-speaking attending terms. These models permit investigation as to whether the attending effect is stronger or weaker in the presence or in the absence of enhanced interpreter services.

Because satisfaction variables were sums of Likert-scale responses and patients, in general, reported a high level of satisfaction, the resulting scores were not normally distributed. We modeled them with ordinal probit regression. ${ }^{21}$ The regression coefficients comparing groups have the convenient interpretation as adjusted "effect sizes" for latent satisfaction, i.e., the mean difference between groups, divided by the within-group standard deviation. For the number of ED visits, number of hospital readmissions, LOS, and numbers of radiology tests and specialty consultations, we used Poisson regression. The regression coefficients, when exponentiated, are interpreted as adjusted relative values of the mean response (e.g., number of hours in hospital, number of tests) comparing 1 group to another. We employed robust standard errors to protect against incorrect variance assumptions. ${ }^{22}$ For binary adherence to follow-up, we used logistic regression. Analyses were conducted using STATA, v.9.0.

\section{RESULTS}

The sample included 323 adult inpatients: 124 Spanishspeakers whose physicians had access to the intervention (of 148 eligible and approached; SS-I), 99 Spanish-speakers whose physicians only had access to usual interpreter services (of 144 eligible and approached; SS-U), and 100 Englishspeakers (of 212 eligible and approached; Table 1). There were no significant differences between the SS-I and SS-U groups or between intervention and control firm English-speakers in any sociodemographic characteristic, history of health care utilization, self-rated health, or Charlson cormorbidity index (Table 1). Nevertheless, in the regression models, we adjusted for variables exhibiting modest differences between firms in either the SS and/or the ES groups.

Thirty-two attending physicians cared for patients on the intervention firm and 26 on the usual care firm. Significantly, more attending physicians on the intervention firm were proficient in Spanish $(n=9 ; 28 \%)$ than on the usual care firm $(n=4 ; 15 \%, p<0.001)$. Forty-four residents cared for patients on the intervention firm and 44 on the usual care firm. The firms had similar numbers of residents proficient in Spanish (24 and 26, respectively).

All study participants reported high levels of satisfaction. The SS-I and SS-U groups had similar unadjusted mean nursing (18.8 [SD=3.5] vs 18.6 [SD=3.3]), physician (20.6 [SD $=1.9$ ] vs 20.2 [SD=2.5]), and overall hospital satisfaction scores (13.0 [SD=2.0] vs 13.0 [SD=2.0]). In the adjusted probit regression analyses using English-speaking patients to control for firm effects, there was no significant impact of the intervention on any of the 3 satisfaction scores (Table 2). However, having a Spanish-speaking attending physician positively and significantly impacted Spanish-speaking patients' satisfaction with the doctor and with the hospital stay (Table 2). This effect is more strongly driven by patients whose physicians did not have access to the intervention (Table 3), although the effect of Spanish-speaking attending physician on patient satisfaction was not significantly different when comparing the SS-U and SS-I groups (Table 3).

There was a significant difference in unadjusted mean patient LOS between the SS-I (5.00 days [SD=4.06]) and SS- 
Table 1. Patient Characteristics

\begin{tabular}{|c|c|c|c|c|}
\hline & $\begin{array}{l}\text { Spanish intervention } \\
\text { firm }(n=124)\end{array}$ & $\begin{array}{l}\text { Spanish usual care } \\
\text { firm }(n=99)\end{array}$ & $\begin{array}{l}\text { English intervention } \\
\text { firm }(n=52)\end{array}$ & $\begin{array}{l}\text { English usual care } \\
\text { firm }(n=48)\end{array}$ \\
\hline Age, mean (SD) & $51(16)$ & $47(17)$ & $46(15)$ & $47(12)$ \\
\hline Female (\%) & 50 & 59 & 42 & 52 \\
\hline \multicolumn{5}{|l|}{ Ethnicity (\%) } \\
\hline Mexican & 80 & 87 & 7 & 10 \\
\hline Caribbean & 4 & 3 & 3 & 2 \\
\hline Central/South American & 16 & 10 & 1 & 2 \\
\hline Black & 0 & 0 & 76 & 73 \\
\hline White/other & 0 & 0 & 13 & 13 \\
\hline Years in US, mean (SD) & 13 (13.3) & $12(11.3)$ & NA & NA \\
\hline \multicolumn{5}{|l|}{ Language (\%) } \\
\hline Spanish only & 84 & 83 & 2 & 2 \\
\hline Spanish > English & 16 & 15 & 3 & 2 \\
\hline Both equally & 0 & 2 & 3 & 6 \\
\hline English > Spanish & 0 & 0 & 1 & 2 \\
\hline English only & 0 & 0 & 91 & 88 \\
\hline \multicolumn{5}{|l|}{ Education (\%) } \\
\hline Elementary & 52 & 56 & 6 & 6 \\
\hline Middle school & 11 & 8 & 2 & 4 \\
\hline Some high school & 18 & 17 & 12 & 26 \\
\hline High school or GED & 10 & 10 & 29 & 28 \\
\hline Some or $>$ college & 8 & 9 & 51 & 36 \\
\hline \multicolumn{5}{|l|}{ Income $(\%)$} \\
\hline$<\$ 10,000$ & 60 & 61 & 57 & 45 \\
\hline$\$ 10,000-24,999$ & 24 & 26 & 28 & 36 \\
\hline$\$ 25,000$ or more & 4 & 1 & 15 & 18 \\
\hline Do not know & 12 & 12 & 0 & 1 \\
\hline \multicolumn{5}{|l|}{ Insurance (\%) } \\
\hline None & 89 & 92 & 69 & 66 \\
\hline Public & 6 & 5 & 23 & 25 \\
\hline Private & 1 & 0 & 4 & 7 \\
\hline Other & 4 & 3 & 4 & 2 \\
\hline Seen physician in the last year (\%) & 75 & 68 & 73 & 75 \\
\hline Hospitalized in the last year (\%) & 46 & 38 & 66 & 50 \\
\hline Fair/poor health status (\%) & 76 & 69 & 69 & 75 \\
\hline Charlson comorbidity index, mean (SD) & $1.7(1.9)$ & $1.5(1.8)$ & $1.7(2.1)$ & $1.4(1.7)$ \\
\hline
\end{tabular}

There were no significant differences at $p<0.05$ in the sociodemographic characteristics or health care measures between Spanish-speaking patients on the intervention and usual care firms or between English-speaking patients on the intervention and usual care firms.

$\mathrm{U}$ groups (5.97 days $[\mathrm{SD}=5.31] ; p=0.03$ ). There were no other significant differences between the SS-I and SS-U groups in the unadjusted mean number of radiology tests per person $(2.07[\mathrm{SD}=3.11]$ vs $2.39[\mathrm{SD}=2.73] ; p=0.18)$ or consultations

Table 2. Impact of Interpreter Service Intervention and Spanishspeaking Attending Physician on Satisfaction Among Spanishspeaking Patients

\begin{tabular}{llc}
\hline \hline & Intervention* & $\begin{array}{l}\text { Spanish-speaking } \\
\text { attending }^{\dagger}\end{array}$ \\
\hline $\begin{array}{l}\text { Satisfaction } \\
\text { with nursing }\end{array}$ & $-0.41(-0.97,0.15)$ & $0.12(-0.23,0.48)$ \\
$\begin{array}{c}\text { Satisfaction } \\
\text { with physicians }\end{array}$ & $-0.31(-0.90,0.29)$ & $0.42(0.03,0.81)$ \\
$\begin{array}{c}\text { Satisfaction with } \\
\text { hospital stay }\end{array}$ & $-0.48(-1.1,0.13)$ & $0.55(0.12,0.99)$ \\
\hline
\end{tabular}

Three separate regression models, each including controls for gender, age, racial/ethnic identification, education, marital status, number of times seen by a physician in the past year, number of hospitalizations in the last year, self-rated health, and CCI score.

Figures are probit regression coefficients (95\%CIs), which are equivalent to effect sizes for:

*The difference between SS-I and SS-U groups attributable to interpreter services intervention, in units of within-group standard deviation, controlling for Spanish-speaking attending.

${ }^{\dagger}$ The difference between Spanish-speaking patients with and without a Spanish-speaking attending, controlling for physician team and interpreter services intervention. per person $(0.46[\mathrm{SD}=0.63]$ vs $0.58[\mathrm{SD}=0.66] ; p=0.17)$ while hospitalized; $\mathrm{ED}$ visits $(0.15[\mathrm{SD}=0.47]$ vs 0.08 [SD=0.37]; $p=0.06)$; or hospitalizations $(0.34[\mathrm{SD}=0.80]$ vs $0.35[\mathrm{SD}=$ 0.71 ; $p=0.70$ ) per person in the 3 months after discharge; or percentage of patients adhering to follow-up $168 \%$ vs $69 \%$; $p=0.82$ ). In the adjusted Poisson regression analyses, again using the English-speaking patients to control for firm effects,

Table 3. Impact of Spanish-speaking Attending on Spanishspeaking Patients' Satisfaction in the Intervention and Usual Care Groups

\begin{tabular}{lll}
\hline \hline & $\begin{array}{l}\text { Intervention } \\
\text { group }\end{array}$ & $\begin{array}{l}\text { Usual care } \\
\text { group }\end{array}$ \\
\hline $\begin{array}{c}\text { Satisfaction } \\
\text { with nursing }\end{array}$ & $0.05(-0.59,0.69)$ & $0.16(-0.27,0.58)$ \\
$\begin{array}{c}\text { Satisfaction } \\
\text { with physicians }\end{array}$ & $0.34(-0.13,0.80)$ & $0.62(-0.11,1.4)$ \\
$\begin{array}{c}\text { Satisfaction with } \\
\text { hospital stay }\end{array}$ & $0.51(0.00,1.02)$ & $0.67(-0.19,1.5)$ \\
\hline
\end{tabular}

Three separate regression models, each including controls for gender, age, racial/ethnic identification, education, marital status, number of times seen by a physician in the past year, number of hospitalizations in the last year, self-rated health, CCI score, and physician team.

Figures are probit regression coefficients $(95 \%$ CIs), which are equivalent to effect sizes for difference between Spanish-speaking patients with and without a Spanish-speaking attending, stratified by receipt of interpreter services intervention. 
there was no significant impact of the intervention on mean LOS, number of ED visits or hospitalizations after discharge, radiology tests or consultations while hospitalized (Table 4). Similarly, there was no significant impact of the intervention on adherence to follow-up in the adjusted logistic regression analyses using English-speaking patients to control for firm effects (Table 4). Having a Spanish-speaking attending also showed no impact on utilization outcomes, except in the case of ED visits. Having a Spanish-speaking attending significantly reduced the number of $\mathrm{ED}$ visits after discharge for Spanishspeaking patients in both firms ( $p=0.03$; Table 4). Expected adjusted ED visits per Spanish-speaking patient with a Spanish-speaking attending were 0.034 visit/patient compared to 0.166 visit/patient for those without a Spanishspeaking attending. We were unable to assess whether or not this impact was different in the SS-I versus SS-U groups because there were no ED visits for the patients in the SS-I group who had a Spanish-speaking physician.

We could not evaluate the cost-savings of the enhanced interpreter intervention, as the intervention did not significantly impact any hospital or post discharge service utilization. The cost of the enhanced interpreter intervention was \$234/ person in the intervention group $(\$ 34,581$ for 148 eligible SS-I patients). There was a significant reduction of ED visits for Spanish-speaking patients who had a Spanish-speaking physician. Comparing the expected adjusted ED visits per Spanishspeaking patient with (0.034 visit/patient) and without $(0.166$ visit/patient) a Spanish-speaking attending using the model in Table 4, this represents a cost-savings of \$92.02 (\$700.03/ visit $\times 0.131$ visit) per Spanish-speaking patient in the study. There was no additional cost of hiring or retaining Spanishspeaking attending physicians at the study institution, so these savings came without additional expenditure.

\section{Discussion}

We found no significant impact of the enhanced interpreter service intervention on any of our measured outcomes

Table 4. Impact of Interpreter Service Intervention and Spanishspeaking Attending Physician on Length of Stay and Health Care Utilization Outcomes among Spanish-speaking Patients

\begin{tabular}{lll}
\hline \hline & Intervention* & $\begin{array}{l}\text { Spanish-speaking } \\
\text { attending }^{\dagger}\end{array}$ \\
\hline Length of stay & $1.00(0.72,1.42)$ & $0.90(0.75,1.08)$ \\
Consults & $1.24(0.64,2.41)$ & $0.85(0.55,1.30)$ \\
Radiology tests & $1.46(0.90,2.35)$ & $0.96(0.71,1.29)$ \\
ED visits after discharge & $3.09(0.81,11.7)$ & $0.21(0.05,0.86)$ \\
$\begin{array}{l}\text { Hospitalizations } \\
\text { after discharge }\end{array}$ & $0.55(0.15,2.00)$ & $0.97(0.46,2.02)$ \\
$\begin{array}{l}\text { Adherence to follow-up } \\
\text { appointments }\end{array}$ & $0.99(0.18,4.6)$ & $0.95(0.43,2.1)$ \\
\hline
\end{tabular}

Separate regression models, each including controls for gender, age, racial/ethnic identification, education, marital status, number of times seen by a physician in the past year, number of hospitalizations in the last year, self-rated health, and CCI score.

*Ratio of mean values for each outcome, measuring the difference between the SS-I and SS-U groups attributable to interpreter services intervention, controlling for Spanish-speaking attending.

${ }^{\dagger}$ Ratio of mean values for each outcome, comparing Spanish-speaking patients with and without a Spanish-speaking attending, controlling for physician team effects and interpreter services intervention.

${ }^{\ddagger}$ Logistic regression controlling for variables as described above; effects are odds ratios rather than ratios of means for Spanish-speaking patients. However, having a Spanishspeaking physician improved Spanish-speaking patients' satisfaction with physician care and with the hospital stay overall. This increase in satisfaction was more pronounced among patients admitted to the usual care firm, suggesting that the enhanced interpreter services intervention did have an important impact on patients' satisfaction with physician communication and hospital stay. In addition, having a Spanish-speaking attending significantly reduced the number of ED visits Spanish-speaking patients had after discharge.

Whereas the study did not demonstrate that providing enhanced interpreter services results in cost-savings, it provides information on the cost of enhanced interpreter services in the context of a hospital stay. Using the mean LOS (5 days) of Spanish-speakers and mean cost of 1 day $(\$ 2,900)$ in the study hospital, we found that the cost of enhanced interpreter services represents $1.5 \%$ of the overall cost of patient care. This relatively small expenditure for enhanced language access services is very similar in magnitude to that found in an Office of Management and Budget report in 2002. ${ }^{9}$ The per patient cost of this service is likely an overestimate, as cost would have been lower were the intervention not restricted to a small set of patients; in practice, the 2 study interpreters could have served many more patients. Our study also found that Spanish-speaking attending physicians reduced costs of care by lowering return ED visits without additional expenditure by the study hospital. There may be a cost to other hospitals, however, of providing Spanish-speaking providers as some institutions pay a signing bonus or provide additional salary to bilingual physicians.

There are a number of reasons why we may not have found a clear impact of the enhanced interpreter intervention on the cost and quality of hospital care. First, large firm effects may be masking the effect of the enhanced interpreter intervention. Second, this study compared enhanced interpreter services available 8 hours a day with usual care that included the hospital interpreter services available 10 hours a day. Whereas we know that our enhanced interpreter services were superior to the hospital interpreter services in interpreter training and accessibility, it may be that the enhanced services were not accessed more frequently than the usual hospital services, blunting the impact of the intervention. It is also possible that the intervention influenced nurse practice since the firms are not constrained to caring for patients on specific medicine wards, thus all nurses were exposed to the intervention. However, it is unlikely that a nurse crossover effect limited our ability to detect a difference as a result of the intervention because, anecdotally, the nurses were not frequent users of the enhanced interpreter services. Third, a large proportion of attending and resident physicians at the study institution is fluent in Spanish. It may be that the interpreters did not add to the impact of attending and resident physicians who were already providing language access in both firms. Fourth, our interpreter service intervention may not have been robust enough. Interpreters were only available 8 hours/day and their use depended on nurses, physicians, and other staff voluntarily calling for their services or patients' requesting the services verbally or through a bedside card. Greater integration of interpreter services into the care routine may have had a greater impact. Interpreters may not have as great an impact on a hospital stay where patients are closely monitored over time versus a setting like an $\mathrm{ED}$ where physicians have to make decisions about whether or not it is safe to release a 
patient. Finally, the satisfaction measure we used may not have been sensitive enough to measure the impact of the interpreter services on communication in the hospital.

In addition to these limitations, this study has several strengths. First, we included English-speakers in our study to control for firm effects and thus isolate the impact of our intervention on satisfaction and hospital service utilization. If we had not done so, we would have erroneously concluded that the enhanced interpreter service intervention reduced the LOS instead of identifying this difference as a result of firm differences. Second, whereas not a randomized controlled trial, the assignment of the patients in the study to firm was independent of patient or firm characteristics, resulting in study groups that were very similar across sociodemographic and other characteristics. Third, we measured physician Spanish language fluency. This not only allowed us to control for the impact of physician language concordance on our study outcomes, it provided us with the only data we are aware of on how Spanish language concordance between physicians and patients impacts hospital service utilization and cost.

These strengths allowed us to provide useful information on the cost of providing enhanced inpatient interpreter services relative to the total cost of a hospital stay. We consider $\$ 240$ a small price to pay to ensure that hospital professional staff are able to provide the standard of care to all patients-to be able to understand and speak to them. The fact that we did not see an impact of this enhanced communication on our chosen outcome measures does not mean that interpreters are not essential to the provision of high quality care to patients with limited English proficiency. It indicates that more research needs to be done in settings where language concordant physicians and other linguistic access services are not as readily available as they were in our study setting. It also highlights the valuable contribution bilingual physicians make to the provision of health care in our increasingly diverse society, underscoring the importance of efforts to increase the ethnic and linguistic diversity of the medical profession.

Acknowledgments: This study was made possible by a contract with the Office of Minority Health (OMH), Department of Health and Human Services. We are indebted to Betty Hawks and Valerie Welsh at $\mathrm{OMH}$ and Angela Ware, Ph.D. from COSMOS Corporation for their support and feedback and to the expert panel they convened. We would also like to acknowledge all the study staff: Eduardo Encinas, Yolanda Diaz, Mayank Gupta, Veronica Hernandez, Gabriela Juarez-Dominguez, Lilia Lazaro, David Moreno, Lisa Stevak, and Shwetha Sungay. We must also thank Rebecca Roberts, M.D. for her help with obtaining information regarding hospital costs. Results were presented at the Office of Minority Health Summit, January 2006 and the 29th annual meeting of the Society of General Internal Medicine, May 2006.

Conflict of Interest: None disclosed.

Corresponding Author: Elizabeth A. Jacobs, MD, MPP; Collaborative Research Unit, John H. Stroger Jr. Hospital of Cook County,
1900 West Polk Street, 16th Floor, Chicago, IL 60612-9985, USA (e-mail: ejacobs@rush.edu).

\section{REFERENCES}

1. U.S. Census Bureau. DP-2. Profile of selected social characteristics: 2000. Available at: http://www.census.gov/Press-Release/www/2002/ dptables/2k48.xls. Accessed March 17, 2003.

2. Woloshin S, Bickell NA, Schwartz LM, Gany F, Welch G. Language barriers in medicine in the United States. JAMA. 1995;273:724-8.

3. Association of State and Territorial Offices. ASTHO Bilingual Health Initiatives: Report and Recommendations. Washington DC: Office of Minority Health; 1992.

4. Anonymous. Need for interpreter/translation services critical in hospitals and other clinical settings. Health Care Strateg Manage. 1995;13:15.

5. Baker DW, Parker RM, Williams M, Coates W, Pitkin K. Use and effectiveness of interpreters in an emergency department. JAMA. 1996;275:783-8.

6. Ginsberg C, Martin D, Andrulis Y, Shaw-Taylor, McGregor C. Interpretation and Translation Services in Health Care: A Survey of US Public and Private Teaching Hospitals. Washington DC: National Public Health and Hospital Institute; 1995.

7. Schmidt R, Ahart A, Schur G. Limited English Proficiency as a Barrier to Health and Social Services. Washington DC: Macro International; 1995.

8. Press Release: New survey shows language barriers causing many Spanishspeaking Latinos to skip care. December 12, 2001. Available at: http://www. hablamosjuntos.org/mediacenter/pressreleases/default.12122001.asp. Accessed August 22, 2007.

9. Graham J. Assessment of cost and benefits associated with implementation of Executive Order 13166. Fed Regist. 2001;66(231):59824-5.

10. Radcliffe Anderson E. Comments on policy guidance on the prohibition against national origin discrimination as it affects persons with limited English proficiency. Written communication to Carol Brown, Office of Civil Rights, Oct 24;2001.

11. Drennan G. Counting the costs of language services in psychiatry. S Afr Med J. 1996;86:343-5.

12. Rader GS. Management decisions: Do we really need interpreters? Nurs Manage. 1988;19:46-8.

13. Hampers LC, Cha S, Gutglass DJ, Binns HJ, Krug SE. Language barriers and resource utilization in a pediatric emergency department. Pediatrics. 1999; 103:1253-6.

14. Jacobs EA, Shepard DS, Suaya JA, Stone E. Overcoming language barriers in health care: costs and benefits of interpreter services. Am J Public Health. 2004;94:866-9.

15. Hampers LC, McNulty JE. Professional interpreters and bilingual physicians in a pediatric emergency department. Arch Pediatr Adolesc Med. 2002;156:1108-13.

16. Bernstein J, Bernstein E, Dave A, et al. Trained medical interpreters in the emergency department: effects on services, subsequent charges, and follow-up. J Immigr Health. 2002;4:171-6.

17. U.S. Department of Health and Human Services. Assessing the Impact of Provider-Patient Language Barriers on Health Care Costs and Quality: Final Report. Rockville MD: U.S. Department of Health and Human Services; 2006, April.

18. Charlson ME, Szatrowski TP, Peterson J, Gold J. Validation of a combined comorbidity index. J Clin Epidemiol. 1994;47:1245-51.

19. Agency for Healthcare Research and Quality. CAHPS hospital survey. Available at: http://www.hcahpsonline.org/surveyinstrument.asp. Accessed August 25, 2006.

20. American Council on the Teaching of Foreign Languages. Oral proficiency interview. Available at: http://www.actfl.org/i4a/pages/index.cfm? pageid=3642\#speaking. Accessed August 25, 2006.

21. Davidson R, MacKinnon JG. Estimation and Inference in Econometrics. New York: Oxford University Press; 1993.

22. White H. A heteroskedasticity-consistent covariance-matrix estimator and a direct test for heteroskedasticity. Econometrica. 1980;48:817-30. 